\title{
Pancreatitis aguda recurrente idiopática. Reporte de caso clínico y revisión de la literatura
}

\section{Reporte de un caso}

Delgado-Sánchez Jonathan Dazaeth*, Herrera-Beiza Bernardo Miguel*, García-Luis Ashley Michelle*, Herrada-Aguilar Cynthia Jazmín*, Lepe-Valadez Priscila Sabrina*, Barba Valadez Claudia Teresa**, López- Rodríguez Jorge Luis***

\section{Resumen}

- Introducción: la pancreatitis aguda recurrente (PAR) es una

- condición clínica caracterizada por episodios repetidos de

- pancreatitis aguda (PA). Las causas más frecuentes son cálcu-

- los biliares y alcoholismo; sin embargo, cuando no se consigue

- determinar la causa, lo cual ocurre entre el $10 \%$ al 25\% de los

- pacientes, se determina como pancreatitis aguda recurrente

- idiopática (PARI). Caso clínico: se presenta el caso de una pa-

- ciente del sexo femenino de 32 años con dos episodios de PA,

- con antecedente de colecistectomía laparoscópica. La pacien-

- te presentó nuevo episodio de PAR grave que requirió manejo

- intrahospitalario. Durante el abordaje diagnóstico se identificó

- como causa microlitiasis, por lo que se realizó, unos meses

- después del cuadro agudo, una colangiopancreatografía re-

- trógrada endoscópica (CPRE) y esfinterotomía. Conclusión: la

- pancreatitis aguda recurrente idiopática tiene como factores

- causales poco frecuentes a la microlitiasis, hipercalemia, hiper-

- trigliceridemia y la inducción por medicamentos. LUXMÉDICA,

- ANO 15, NÚMERO 44, MAYO-AGOSTO 2020, PP 39-47.

-

- Palabras clave: pancreatitis aguda (PA), pancreatitis aguda re- currente (PAR), pancreatitis aguda recurrente idiopática (PARI)

\section{Abstract}

Introduction: Recurrent acute pancreatitis (RAP) is a clinical condition characterized by repeated acute pancreatitis (AP). The most frequent causes are gallstones and alcoholism. However, when the cause cannot be determined, which occurs in 10\% to $25 \%$ of patients, it is known as idiopathic recurrent acute pancreatitis (IRAP). Caso report: We present a 32-year-old female patient with two episodes of AP, with a history of laparoscopic cholecystectomy. The patient presented a new episode of severe RAP that required in-hospital treatment. Microlithiasis was identified as the cause, and an endoscopic retrograde cholangiopancreatography (ERCP) and sphincterotomy were performed a few months after the acute condition. Conclusion: Microlithiasis, hyperkalemia, hypertriglyceridemia, and drug-induction are uncommon causes of idiopathic recurrent acute pancreatitis. LUXMÉDICA, AÑO 15, NÚMERO 44, MAYO-AGOSTO 2020, PP 39-47.

Keywords: acute pancreatitis, recurrent acute pancreatitis, idiopathic recurrent acute pancreatitis 


\section{Introducción}

La pancreatitis aguda (PA) es un proceso inflamatorio del páncreas, que generalmente cursa con una glándula morfológicamente funcional. Se caracteriza por cambios edematosos autolimitados del páncreas y puede tener repercusión sistémica. ${ }^{1-3}$ Posterior al ataque inicial, aproximadamente del $11 \%$ al $32 \%$ de los pacientes evolucionan con ataques recidivantes, ${ }^{4-6}$ dependiendo de la causa. ${ }^{1}$ La pancreatitis aguda recurrente (PAR), inicialmente reconocida como entidad clínica en la clasificación de Marsella, ${ }^{7}$ se caracteriza por episodios repetitivos de PA. La dificultad para diferenciarla de la pancreatitis crónica (PC) se estableció en la clasificación de TIGAR-O, ya que los episodios repetitivos pueden ocasionar daño crónico. ${ }^{4}$ Gullo y cols. mostró que el $10 \%$ de los pacientes con PA y un $26 \%$ con PAR evolucionan a pancreatitis crónica. ${ }^{5}$ La presencia de cálculos biliares y el consumo de alcohol se encuentran como las principales etologías de la PAR. La pancreatitis aguda recurrente idiopática (PARI) se diagnostica si no se identifica una causa definitiva de PAR después de las investigaciones estándar.

Es necesario un abordaje clínico profundo empezando con el historial médico de un paciente para determinar el factor etiológico y proveer de un tratamiento médico oportuno y, por ende, prevenir otro ataque. Sin embargo, en grupos con PARI existe la controversia de las causas poco comunes que pueden definir el manejo terapéutico. El propósito de este caso clínico es exponer la importancia de definir correctamente PAR y PARI para establecer un diagnóstico y tratamiento oportuno, una vez identificados los factores etiológicos.

\section{Presentación del caso clínico}

Mujer de 32 años, con el antecedente de dos episodios de PA, 11 y 6 años antes del cuadro actual, incluso había sido sometida a colecistectomía por vía laparoscópica. No se identificaron factores de riesgo como toxicomanías, ingesta de medicamentos, o traumatismos. El diagnóstico de PA se estableció mediante criterios clínicos, de laboratorio y gabinete, y se hospitalizó para tratamiento médico y nutricional. No se documentaron fallas orgánicas, la evolución fue satisfactoria y fue egresada a su domicilio con tratamiento médico a base de soluciones parenterales, analgésicos, antimicrobianos y tubo nasoenteral (TNE), con fórmula inmunomoduladora (Inmunex®) a infusión continua, calculada a $28.8 \mathrm{kcal} / \mathrm{kg} /$ día y proteínas $1.06 \mathrm{gr} / \mathrm{kg} /$ día.

En la consulta inicial, realizada en su domicilio, se le encontró con una frecuencia cardiaca 100 latidos por minuto, palidez de tegumentos y en la exploración físi$\mathrm{ca}$, la presencia de una colección palpable y dolorosa en el flanco izquierdo y en la fosa iliaca izquierda, por lo que se decidió hospitalizar. A su ingreso, se encontró un índice de masa corporal (IMC) de 29.7 kg/m2, frecuencia cardiaca de 96x', presión 
arterial de $110 / 70 \mathrm{~mm}$ de $\mathrm{Hg}$, frecuencia respiratoria de 18 por minuto y una temperatura de $37^{\circ} \mathrm{C}$. Se auscultó una hipoventilación en la base pulmonar izquierda y dolor abdominal con aumento de volumen en el flanco y fosa iliaca izquierda, sin datos de irritación peritoneal. Al día siguiente inició con fiebre intermitente y la biometría hemática de control, practicada el día anterior, mostró leucocitosis de 12000, por lo que se agregó ciprofloxacino al tratamiento. Un día después, presentó un episodio de bacteremia caracterizado por escalofríos, fiebre, sudoración y taquicardia. Por este motivo se practicó una tomografía axial computarizada (TAC) dinámica de control, que reveló datos en relación con la pancreatitis Balthazar $\mathrm{E}_{\text {, }}$ con necrosis menor del 30\% de la extensión del páncreas, que tiene un Índice de severidad tomográfica de 6 puntos (figura 1).
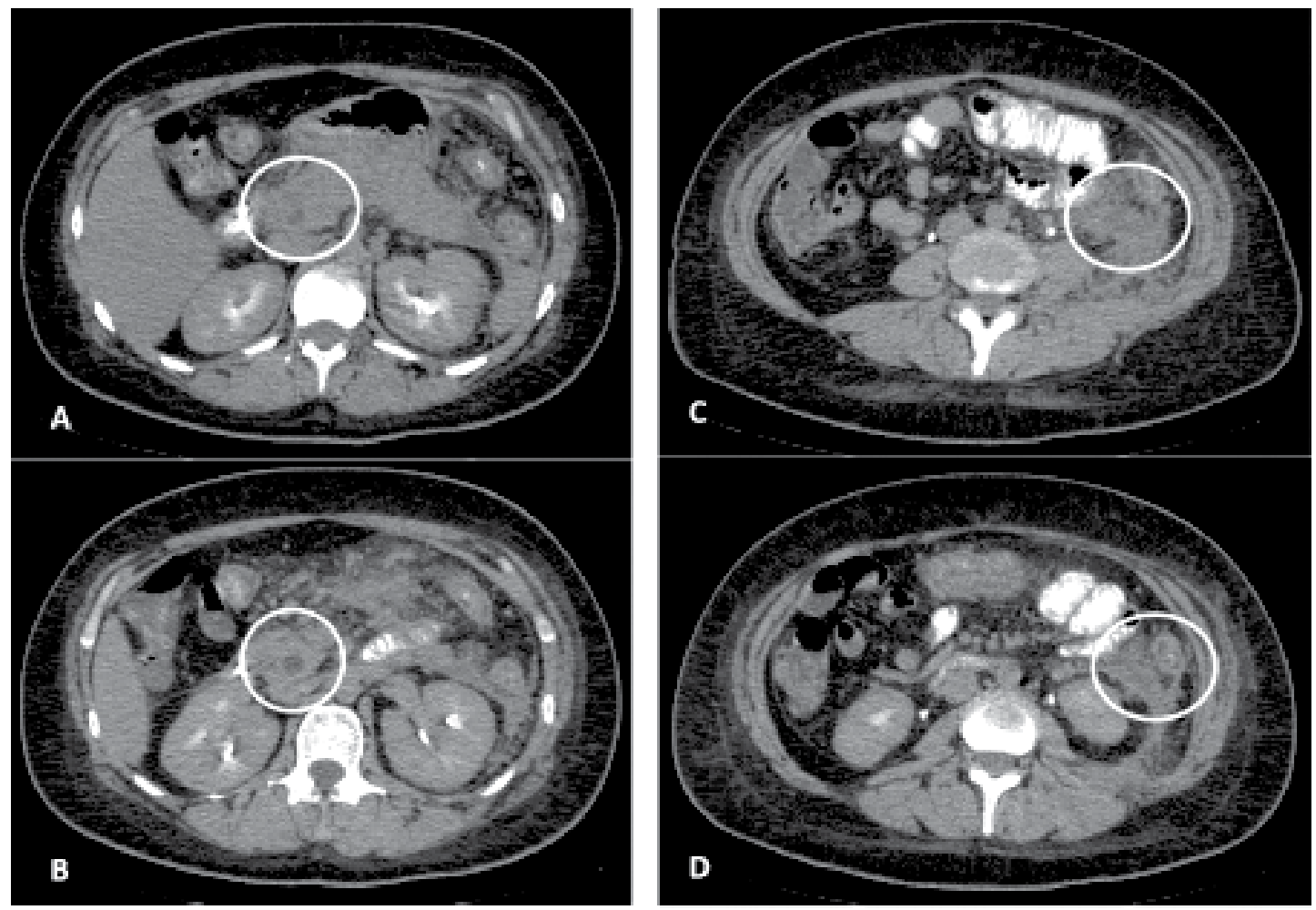

Fig. 1. Corte axial de tomografía computarizada contrastada de abdomen. En los círculos de color blanco se observa en $A$ y $B$ un aumento de las dimensiones del páncreas, heterogéneo de forma difusa. Zonas hipodensas mal delimitadas en el cuerpo y en la cabeza con densidades que sugieren necrosis. Mientras que en los círculos blancos de $C$ y $D$ se aprecia una colección peripancreática delimitada por el espacio pararrenal anterior, el espacio esplenorrenal, llega hasta la fascia lateroconal, contacta con el músculo psoas y se dirige hacia el fondo de saco de Douglas. 
Se le inició tratamiento con nutrición enteral por sonda nasoyeyunal, con dieta inmunomoduladora (Inmunex ${ }^{\circledR}$ ) a dilución normal y a infusión continua; soluciones parenterales, inhibidor de la bomba de protones, analgésicos y doble esquema de antibiótico, medidas de vigilancia y control metabólico (hiperglicemia), respiratorio y de trombosis venosa profunda. Después de 10 días de estancia hospitalaria fue egresada a su domicilio, continuó con soporte nutricio por vía en- teral retirada tres días más tarde y la dieta oral se indicó baja en grasas, alta en proteínas y carbohidratos.

Ya que existía la necesidad de identificar etiología biliar del episodio reciente de $P A$, se practicó una CPRE, la cual mostró una papila desfloreada, sin litos evidentes en la vía biliar. Se obtuvo una muestra de bilis para cristalografía y se realizó una esfinterotomía. En la cristalografía se reportó elementos morfológicamente compatibles con cristales de colesterol (Fig. 2).

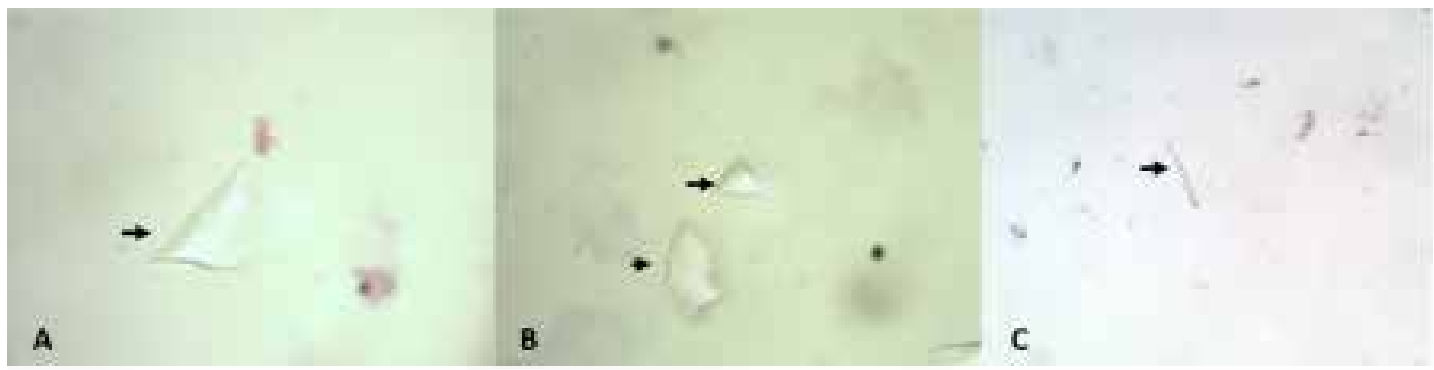

Fig. 2. A/B/C: Cristalografía. Se aprecia sobre un fondo proteináceo estructuras morfológicamente compatibles con cristales de colesterol con forma de rombo (flechas negras en A y B) y "agujas" (flecha negra en $C$ ).

\section{|||||||||||||||||||||||||||||||||||||||||||||||||||||||||||||||||||||||||||||||||||||||||||||||||||||||||||||||||||||||||}

\section{Discusión}

Se podría definir la PAR como la existencia de dos o más episodios de pancreatitis aguda ${ }^{4}$ con dos o tres de los siguientes elementos: (a) dolor abdominal sugestivo de pancreatitis (dolor epigástrico frecuente e irradiado a la espalda); (b) análisis serológicos o niveles de lipasa; (c) hallazgos característicos en tomografía computarizada (TC), resonancia magnética (RM) o ultrasonido abdominal (UA). ${ }^{3,8}$ Estos criterios se pueden encuadrar en el caso presentado; sin embargo, al no determinar la etiología o los factores de riesgo, la paciente reincidió con un nuevo ataque de PA. Para evitar la ambigüedad diagnóstica entre PA, PAR y PC, en el 2018 la Recurrent Acute Pancreatitis International State-of-the-Science Conference With Recommendations, ${ }^{3,9}$ concretó una nueva definición como un síndrome de múltiples respuestas inflamatorias agudas distintas que se originan dentro del páncreas en individuos con factores genéticos, ambientales, traumáticos, morfológicos, metabólicos, biológicos u otros factores de riesgo que experimentaron dos o más episodios de AP documentada y separados por al menos tres meses. ${ }^{3}$

Un "evento centinela" es el primer evento de PA. Cualquier factor con la capacidad de desencadenar un episodio inicial de PA puede provocar episodios recurrentes ${ }^{4}$ a no ser que el factor causante 
sea identificado y tratado correctamente. PAR puede ser el resultado de la contribución de múltiples factores y su posible infección. El factor etiológico más frecuente en PAR es la obstrucción biliar, siendo la causa principal la enfermedad litiásica en un $40 \% .^{2,10-12}$ El etilismo representa un $30 \%$, el tabaco es un factor dosis-dependiente independiente para PAR y está relacionado con la evaluación a una PC. 3,13,14

El rol de la genética ha ganado fuerza en las últimas décadas, las pruebas genéticas (mutaciones en CFTR, SPINK1, PRSS1, CTRC y otros genes) están indicadas en la mayoría de los pacientes más jóvenes ( $<35$ años). ${ }^{3}$

Entre el $10 \%$ al $25 \%$ de los casos, ${ }^{3,6,13}$ la etiología es desconocida aún después de una búsqueda minuciosa, entonces se define como pancreatitis aguda recurrente idiopática (PARI). Entre las causas menos comunes de PARI, están la PAR autoinmune $(<5 \%)$, microlitiasis, (6$10 \%)^{3,13,15}$ y factores metabólicos como hipercalcemia e hipertrigliceridemia. Las causas controversiales de PARI son las anormalidades anatomo-funcionales: (a) páncreas divisum, que es una la anormalidad congénita más común del páncreas (514\%) y tiene una prevalencia del $26 \%$ como posible factor; (b) disfunción del esfínter de Oddi (30-36\%). ${ }^{16}$ En la tabla 1 se muestran los factores etiológicos con sus respectivas pruebas diagnósticas.

\section{Tabla I}

\section{Pruebas diagnósticas para RAP dependiendo la etiología específica ${ }^{6}$}

\begin{tabular}{|c|c|c|c|}
\hline \multicolumn{2}{|c|}{ Etiología } & Prueba diagnostica & Comentario \\
\hline \multirow{2}{*}{ Biliar } & $\begin{array}{l}\text { Cálculos } \\
\text { biliares }\end{array}$ & \multirow{2}{*}{$\begin{array}{l}\text { UA } \\
\text { USE } \\
\text { Microscopia biliar }\end{array}$} & \multirow{2}{*}{$\begin{array}{l}\text { Las enzimas hepáticas elevadas o la } \\
\text { bilirrubina dentro de los } 2 \text { o } 3 \text { días de } \\
\text { la pancreatitis aguda que se resuelve } \\
\text { a la normalidad en unos pocos días es } \\
\text { una pista de pancreatitis biliar. }\end{array}$} \\
\hline & Microlitiasis & & \\
\hline \multicolumn{2}{|c|}{ Hipercalcemia } & $\begin{array}{l}\text { Nivel de calcio sérico }>10.7 \mathrm{mg} / \\
\mathrm{dl}\end{array}$ & $\begin{array}{l}\text { La evaluación paratiroidea es necesa- } \\
\text { ria en la ausencia de otra etiología }\end{array}$ \\
\hline \multicolumn{2}{|c|}{ Hipertrigliceridemia } & Tiroglobulina sérica >100mg/dl & \\
\hline \multicolumn{2}{|c|}{ Tumores pancreatobiliares } & $\begin{array}{l}\mathrm{TC} / \mathrm{RM} \\
\text { USE }\end{array}$ & $\begin{array}{l}\text { Los pacientes con edades entre } 9 \text { y } \\
50 \text { años deben someterse a imágenes } \\
\text { para descartar un tumor pancreatobi- } \\
\text { liar }\end{array}$ \\
\hline \multicolumn{2}{|c|}{ Páncreas divisum } & $\begin{array}{l}\text { USE } \\
\text { CRM }\end{array}$ & $\begin{array}{l}\text { El páncreas divisum por sí solo pue- } \\
\text { de no causar PAR pero puede ser un } \\
\text { cofactor junto con la susceptibilidad } \\
\text { genética }\end{array}$ \\
\hline \multicolumn{2}{|c|}{ Disfunción del esfínter de Oddi } & Manometría del esfínter de Oddi & $\begin{array}{l}\text { Causa controvertida de PAR } \\
\text { Debe sospecharse después de excluir } \\
\text { otras etiologías. } \\
\text { La esfinterotomía biliar con o sin es- } \\
\text { finterotomía pancreática puede bene- } \\
\text { ficiar }\end{array}$ \\
\hline \multicolumn{2}{|c|}{$\begin{array}{l}\text { Anormalidades de la unión pan- } \\
\text { creatobiliar }\end{array}$} & CRM & Causa controversial de PAR \\
\hline \multicolumn{2}{|c|}{ Colédococele } & $\begin{array}{l}\text { CRM } \\
\text { USE }\end{array}$ & $\begin{array}{l}\text { Esfinterotomía es beneficiosa en caso } \\
\text { de episodios dolorosos recurrentes }\end{array}$ \\
\hline
\end{tabular}




\begin{tabular}{|l|l|l|}
\hline Páncreas anular & $\begin{array}{l}\text { TM } \\
\text { USE }\end{array}$ & $\begin{array}{l}\text { Causa controversial de PAR } \\
\text { Factores genéticos }\end{array}$ \\
\hline Autoinmune & $\begin{array}{l}\text { Niveles de IgG4, TC, CRM, USE, } \\
\text { P-ANCA, C-ANCA }\end{array}$ & $\begin{array}{l}\text { La pancreatitis autoinmune es una } \\
\text { causa rara de PAR (más común con el } \\
\text { tipo 2 que con el tipo 1) } \\
\text { La terapia inmunosupresora puede } \\
\text { ser beneficiosa en PAR autoinmune y } \\
\text { vasculitis. }\end{array}$ \\
\hline
\end{tabular}

*Ultrasonido abdominal (US); Ultrasonido endoscópico (USE); Resonancia magnética (RM); Tomografía computarizada (TC); Colangiopancreatografía por resonancia magnética (CRM);

En el caso que presentamos, el estudio de identificación de la causa biliar se efectuó mediante cristalografía, a falta de un ultrasonido endoscópico (USE), y se identificaron los microlitos de colesterol como la causa etiológica. Este seguimiento se contrasta con el algoritmo de la figura 3. Los microlitios son cálculos biliares $<3 \mathrm{~mm}$ de tamaño y no se pueden visualizar en la ecografía abdominal. Sin embargo, hasta el $75 \%$ de los pacientes con PARI y sospecha de micolitiasis desarrollan cálculos biliares en la UA. La micriolitiasis se diagnostica mejor en USE con una sensibilidad del $96 \% .{ }^{16,17}$ También se puede detectar con microscopia biliar con una sensibilidad del $65-95 \% ; i^{18}$ este método es de importancia porque muestra cristales de colesterol. En una paciente con PARI, como la presentada, no alcohólica ni fumadora, deben realizarse las pruebas de gabinete; sin embargo, en nuestro caso no se hizo de esta manera. En la bibliografía se puede encontrar que los cristales de colesterol son evidencia indirecta de microlitiasis biliar. ${ }^{6,13,18,19}$ Los factores metabólicos de la paciente pueden ser parte de una etiología multifactorial desencadenante del curso idiopático. A pesar de que no se determinó la etiología del evento, el procedimiento fue el correcto. El procedimiento sugerido es una colecistectomía pero, tener antecedente de este proceso, el siguiente abordaje es a través de una colangiopancreatografía retrógrada endoscópica (CPRE), ${ }^{20}$ además de que la evidencia ha demostrado la eficacia de una CPRE junto con una esfinterotomía biliar como medida profiláctica para disminuir el riesgo de nuevos episodios de PA. 4,6,18,21-23 


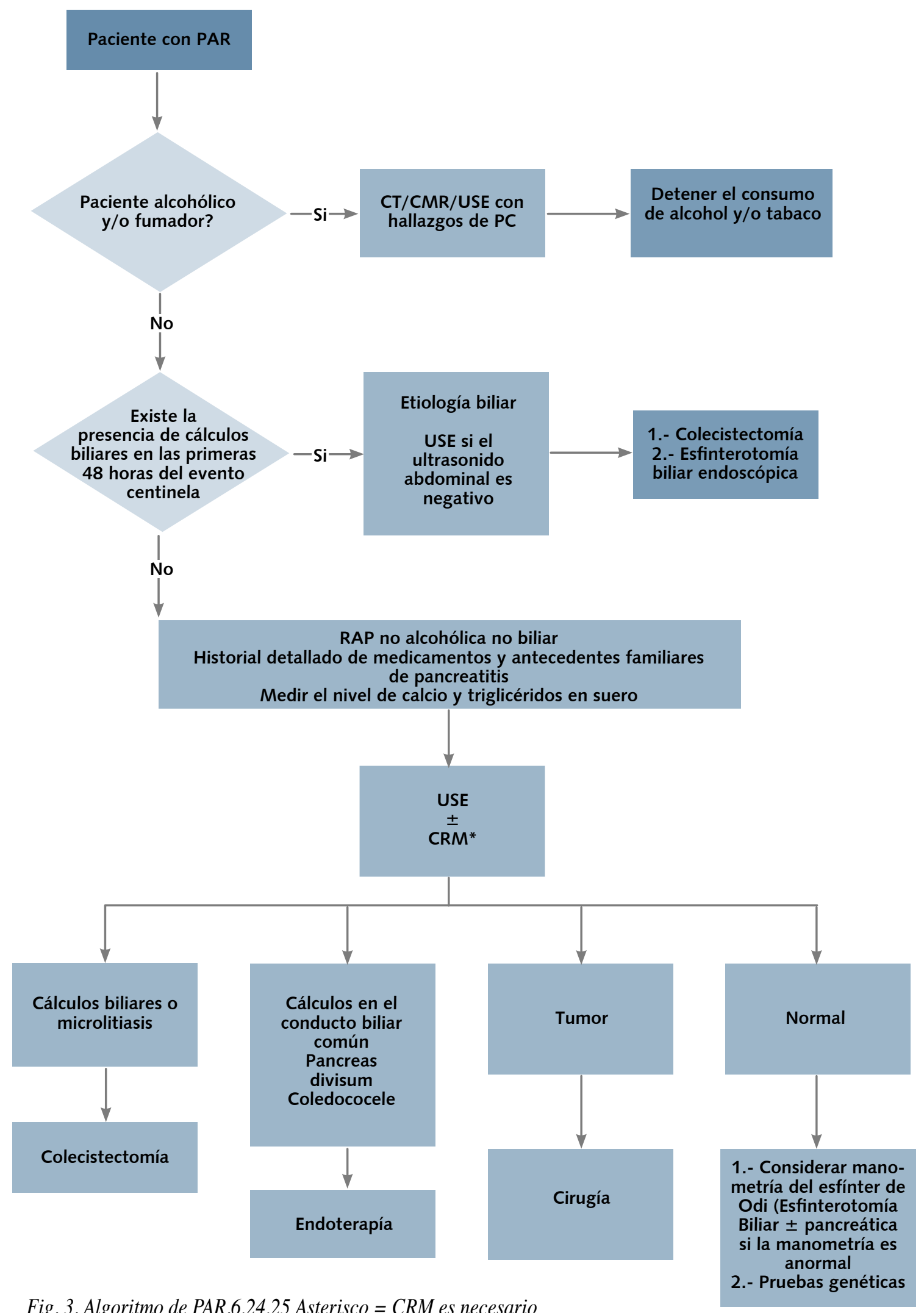

Fig. 3. Algoritmo de PAR.6,24,25 Asterisco $=$ CRM es necesario si los hallazgos de USE son equívocos o negativos. 
La RAPI es un síndrome ambiguo complejo de diagnosticar debido a los síntomas dispersos y poco específicos de cada etiología. Por esto, cobra especial relevancia una historia clínica completa y detallada para detectar de manera oportuna el evento centinela. Pese a que la enfermedad sigue un curso idiopático se puede seleccionar un manejo profiláctico para los pacientes.

\section{Bibliografía}

1. Lankisch PG, Apte $M$, Banks PA. Acute pancreatitis. Lancet [Internet]. 2015 Jul 4;386(9988):85-96. Available from: https://doi.org/10.1016/S01406736(14)60649-8

2. Testoni PA. Acute recurrent pancreatitis: Etiopathogenesis, diagnosis and treatment. World J Gastroenterol [Internet]. 2014 Dec; 20(45):16891-901. Available from: https://www.wjgnet.com/1007-9327/ full/v20/i45/16891.htm

3. Guda NM, Muddana V, Whitcomb DC, Levy $P$, Garg $P$, Cote $G$, et al. Recurrent Acute Pancreatitis. Pancreas [Internet]. 2018 Jul;47(6):653-66. Available from: http://insights.ovid.com/crossref? an=00006676-201807000-00001

4. Sánchez Rodríguez E, García García de Paredes A Albillos A. Manejo actual de la pancreatitis aguda idiopática y la pancreatitis aguda recurrente. Rev Clínica Española [Internet]. 2019 Jun;219(5):266-74. Available from: https://www.revclinesp.es/es-manejo-actual-pancreatitis-aguda-idiopatica-articuloS0014256519300438

5. Gullo L, Migliori M, Pezzilli R, Oláh A, Farkas G, Levy $P$, et al. An Update on Recurrent Acute Pancreatitis: Data From Five European Countries. Am J Gastroenterol [Internet]. 2002;97(8). Available from: https:// journals.Iww.com/ajg/Fulltext/2002/08000/ An_Update_on_Recurrent_Acute_Pancreatitis_. Data.21.aspx

6. Jagannath S, Garg PK. Recurrent Acute Pancreatitis: Current Concepts in the Diagnosis and Management. Curr Treat Options Gastroenterol [Internet]. 2018 Dec 19;16(4):449-65. Available from: http:// link.springer.com/10.1007/s11938-018-0196-9

7. Sarles $H$, Sarles JC, Camatte $R$, Muratore $R$, Gaini $M$, Guien $C$, et al. Observations on 205 confirmed cases of acute pancreatitis, recurring pancreatitis, and chronic pancreatitis. Gut [Internet]. $1965 \mathrm{Dec}$ 1;6(6):545-59. Available from: https://www.ncbi. nlm.nih.gov/pubmed/5857891

8. Reichstein JB, Patel V, Mekaroonkamol P, Dacha S, Keilin SA, Cai $Q$, et al. Practice Patterns and Use of Endoscopic Retrograde Cholangiopancreatography in the Management of Recurrent Acute Pancreatitis. Clin Endosc. 2019 Jul;

9. Cote GA, Yadav D, Abberbock JA, Whitcomb DC,
Sherman S, Sandhu BS, et al. Recurrent Acute Pancreatitis Significantly Reduces Quality of Life Even in the Absence of Overt Chronic Pancreatitis. Am J Gastroenterol. 2018 Jun;113(6):906-12.

10. Magnusdottir BA, Baldursdottir MB, Kalaitzakis $E$, Bjornsson ES. Risk factors for chronic and recurrent pancreatitis after first attack of acute pancreatitis. Scand J Gastroenterol. 2019 Jan;54(1):87-94.

11. Agarwal S, George J, Padhan RK, Vadiraja PK, Behera S, Hasan A, et al. Reduction in mortality in severe acute pancreatitis: A time trend analysis over 16 years. Pancreatology [Internet]. 2016 Mar;16(2):194-9. Available from: https://doi. org/10.1016/j.pan.2016.01.012

12. Coté GA, Imperiale TF, Schmidt SE, Fogel E, Lehman $G, M c H e n r y ~ L$, et al. Similar efficacies of biliary, with or without pancreatic, sphincterotomy in treatment of idiopathic recurrent acute pancreatitis. Gastroenterology [Internet]. 2012;143(6):1502-1509.e1. Available from: http://dx.doi.org/10.1053/j.gastro.2012.09.006

13. Garg PK, Tandon RK, Madan K. Is Biliary Microlithiasis a Significant Cause of Idiopathic Recurrent Acute Pancreatitis? A Long-term Follow-up Study. Clin Gastroenterol Hepatol [Internet]. 2007 Jan;5(1):759. Available from: https://linkinghub.elsevier.com/ retrieve/pii/S1542356506006756

14. Cho JH, Jeong YH, Kim KH, Kim TN. Risk factors of recurrent pancreatitis after first acute pancreatitis attack: a retrospective cohort study. Scand J Gastroenterol. $2019 \mathrm{Dec} ; 1-5$.

15. Guo A, Poneros JM. The Role of Endotherapy in Recurrent Acute Pancreatitis. Gastrointest Endosc Clin N Am [Internet]. 2018 Oct;28(4):455-76. Available from: https://linkinghub.elsevier.com/retrieve/pii/ S1052515718307207

16. Mustafa A, Begaj I, Deakin M, Durkin D, Corless DJ, Wilson $R$, et al. Long-term effectiveness of cholecystectomy and endoscopic sphincterotomy in the management of gallstone pancreatitis. Surg Endosc [Internet]. 2014;28(1):127-33. Available from: https://doi.org/10.1007/s00464-013-3138-6

17. Zakko L, Gardner TB. Endoscopic Management of Recurrent Acute Pancreatitis. Clin Gastroenterol Hepatol. 2019 Oct;17(11):2167-70.

18. Ros E, Navarro S, Bru C, Garcia-Pugés A, Valderrama R. Occult microlithiasis in 'idiopathic' acute 
pancreatitis: Prevention of relapses by cholecystectomy or ursodeoxycholic acid therapy. Gastroenterology [Internet]. $1991 \mathrm{Dec} ; 101(6): 1701-9$. Available from: https://linkinghub.elsevier.com/retrieve/ pii/001650859190410M

19. Elta GH. Sphincter of Oddi dysfunction and bile duct microlithiasis in acute idiopathic pancreatitis. World J Gastroenterol [Internet]. 2008;14(7):1023. Available from: http://www.wjgnet.com/1007-9327/full/ v14/i7/1023.htm

20. Sanchez Rodriguez E, Garcia Garcia de Paredes A, Albillos A. Current management of acute idiopathic pancreatitis and acute recurrent pancreatitis. Rev Clin Esp [Internet]. 2019 Jun;219(5):266-74. Available from: https://www.revclinesp.es/es-manejo-actual-pancreatitis-aguda-idiopatica-articuloS0014256519300438

21. Banks PA, Bollen TL, Dervenis C, Gooszen HG, Johnson $C D$, Sarr $M G$, et al. Classification of acute pancreatitis-2012: revision of the Atlanta classification and definitions by international consensus. Gut [Internet]. 2013 Jan;62(1):102-11. Available from: http://gut.bmj.com/lookup/doi/10.1136/gutjnl-2012-302779
22. Parsi Ma, Stevens T, Dumot Ja, Zuccaro G. Endoscopic therapy of recurrent acute pancreatitis. Cleve Clin J Med [Internet]. 2009 Apr 1;76(4):225-33. Available from: https://www.mdedge.com/ccjm/article/95070/gastroenterology/endoscopic-therapyrecurrent-acute-pancreatitis

23. van Geenen EJM, van der Peet DL, Mulder CJ, Cuesta MA, Bruno MJ. Recurrent acute biliary pancreatitis: the protective role of cholecystectomy and endoscopic sphincterotomy. Surg Endosc [Internet]. 2009 May 6;23(5):950-6. Available from: http:// link.springer.com/10.1007/s00464-009-0339-0

24. Kedia S. Recurrent acute pancreatitis: an approach to diagnosis and management. Trop Gastroenterol [Internet]. 2013 Sep 1;34(3):123-35. Available from: http://www.tropicalgastro.com/articles/34/3/recurrent-acute-pancreatitis.html

25. Somogyi L, Martin SP, Venkatesan T, Ulrich CD. Recurrent acute pancreatitis: An algorithmic approach to identification and elimination of inciting factors. Gastroenterology [Internet]. 2001 Feb;120(3):70817. Available from: https://linkinghub.elsevier.com/ retrieve/pii/S0016508501739674 